\title{
Estrategias para la formación de capacidades en ciencia, tecnología e innovación en educación superior
}

\author{
Olga-Lucía Ostos-Ortiz ${ }^{1}$ \\ Diana María Cuartas-Méndez ${ }^{2}$ \\ Fray Eduardo González-Gil ${ }^{3}$
}

Recibido: 01-02-2020

Aceptado: 01-05-2020

\section{Resumen}

Las tendencias específicas de la disciplina (apps, IoT, web, IA y Mediante el desarrollo de un estudio analítico en la Universidad Santo Tomás se generó un programa institucional para la Formación de Capacidades en Ciencia Tecnología e Innovación (CTel), constituido por ocho estrategias. Las estrategias propuestas corresponden a: grupos de estudio, colectivos académicos, grupos de trabajo, semilleros de investigación, programa institucional de jóvenes investigadores, programa de gestores de investigación, programa institucional de vocaciones científicas y trabajos de grado. El programa permite formar capacidades en ciencia, tecnología e innovación en el $100 \%$ de los estudiantes; articular las funciones universitarias; apropiar la cultura de la investigación y la

\footnotetext{
1. Dirección Nacional de Investigación e Innovación, Universidad Santo Tomás. Correo electrónico: dir.unidadinvestigacion@usantotomas.edu.co

ORCID: https://orcid.org/0000-0002-6477-9872

CvLac: http://scienti.colciencias.gov.co:8081/cvlac/visualizador/generarCurriculoCv. do? cod_rh $=0000260010$
}

2. Docente, Dirección Nacional de Investigación e Innovación, Universidad Santo Tomás. Correo electrónico: dianacuartas@usantotomas.edu.co

ORCID: https://orcid.org/0000-0001-5647-4537

CvLac: https://scienti.minciencias.gov.co/cvlac/visualizador/generarCurriculoCv. do?cod_rh $=0000652008$

3. Vicerrector académico general, Universidad Santo Tomás.

Correo electrónico: viceacadgeneral@usantotomas.edu.co

ORCID: https://orcid.org/0000-0002-7692-1020

CvLac: http://scienti.colciencias.gov.co:8081/cvlac/visualizador/generarCurriculoCv.

do?cod_rh=0001448002 
innovación; fomentar la participación de estudiantes y egresados en el desarrollo de proyectos de investigación e innovación con participación del Estado, el sector productivo y la sociedad civil; consolidar las líneas activas de investigación y los campos de acción institucionales en articulación con los Objetivos de Desarrollo Sostenible (ODS), con participación de estudiantes y egresados, potencializando sus capacidades y habilidades para mejorar su perfil profesional desde la perspectiva de investigación e innovación.

Palabras clave: capacidades, competencias, ciencia, tecnología, innovación, grupos, estudio, tesis, científico, talento, cultura, investigación.

\section{Strategies for capacity building in science, technology and innovation in higher education}

\section{Abstract}

The development of an analytical study at the Santo Tomás University generated a Program for Capacities in Science, Technology and Innovation (CTel) consisting of eight strategies. The proposed strategies correspond to study groups, academic groups, work groups, research hotbeds, an institutional program for young researchers, a program for research managers, an institutional program for scientific vocations and graduate work. The Program enables $100 \%$ of students to train science, technology and innovation skills, articulate substantive and adjective functions, appropriate the culture of research and innovation, encourage the participation of students and graduates in the development of research projects and innovation with the participation of the State, the productive sector and civil society, consolidate the active lines 
of research, the institutional fields of action with the participation of students and graduates, enhancing the capacities and abilities of students and graduates to improve their professional profile from the research and innovation perspective.

Keywords: Capacities, competences. science, technology, innovation, groups, study, thesis, scientist, talent, culture, research.

\section{Introducción}

El desarrollo de capacidades en ciencia, tecnología e innovación (CTel) es fundamental para las instituciones de educación superior. En el presente artículo se presenta el caso de la Universidad Santo Tomás (USTA) y el desarrollo del Programa de Formación de Capacidades en CTel, que permite promover el pensamiento creativo y crítico de los estudiantes en un contexto de comprensión y acción social (Universidad Santo Tomás, 2018).

En este contexto, la Dirección de Investigación e Innovación de la USTA ha creado el Programa Institucional de Formación de Capacidades en CTel para que cada estudiante, docente, administrativo y egresado pueda adquirir y perfeccionar las habilidades y competencias necesarias que le permitan: plantear preguntas; diseñar y ejecutar proyectos de investigación que respondan a intereses, problemas y necesidades socialmente relevantes; desarrollar producción académica en el marco de las buenas prácticas científicas con visibilidad e impacto; utilizar herramientas que faciliten los procesos de investigación e innovación; profundizar en temas de interés mediante el uso de bases bibliográficas especializadas; vincularse en escenarios de innovación para dar respuesta a las necesidades del sector público y productivo; gestionar el conocimiento y su apropiación social; participar en redes académicas y de investigación, y otras competencias que incentivan el gusto por la investigación y fortalecen la creatividad, la cultura de la inves- 
tigación y la innovación (Universidad Santo Tomás - Dirección de Investigación e Innovación, 2019; Colciencias, 2018).

Así mismo, el Programa Institucional de Formación en Capacidades en CTel, en el que participan estudiantes, docentes, administrativos y egresados, articula, como lo propone el modelo de gestión de la universidad, la investigación y la innovación como función sustantiva con las demás funciones universitarias (González, 2018): docencia, proyección social, internacionalización, aseguramiento de la calidad y gestión, con una mirada integral que favorece la formación de vocaciones científicas, de innovación y compromiso social que hagan realidad el principio de responsabilidad social universitaria, fortaleciendo valores y competencias como el trabajo en equipo, la cooperación, la responsabilidad, el respeto, la integridad, el liderazgo, la confianza, la autoestima, entre otros (Universidad Santo Tomás, 2004; Ramírez Martínez, Silva Vivas, Hernández Merchán, López Pachón y Ostos Ortiz, 2018). Por otra parte, las estrategias que componen el programa nutren las líneas activas de investigación de los programas en coherencia con los campos de acción definidos por la USTA: sociedad y ambiente (Ostos-Ortiz y Cortés-Gallego, 2019). Así pues, el programa permite la participación de los estudiantes, que desde el inicio de su carrera académica tienen la oportunidad de vincularse a los grupos de investigación y desarrollar producción académica en el marco del Sistema Institucional de Investigación, Innovación y Creación de la USTA (Universidad Santo Tomás - Dirección de Investigación e Innovación, 2019).

\section{Metodología}

El Programa de Formación de Capacidades en CTel surge en el año 2019 a partir de un proyecto de investigación, para el desarrollo institucional, realizado en el año 2018, cuyo objetivo era que el 100 $\%$ de los estudiantes tuvieran la oportunidad de participar en una o más estrategias de formación durante el desarrollo de su carrera académica. Hasta ese momento la estrategia preponderante era la 
participación de estudiantes en semilleros de investigación, espacios de formación extracurriculares que promueven el gusto por la investigación. Sin embargo, la participación en esta iniciativa era inferior al $30 \%$ de los estudiantes por programa (Universidad Santo Tomás - Dirección de Investigación e Innovación, 2019).

Para cumplir con el objetivo del estudio, se diseñó y ejecutó un programa de estrategias pedagógicas que de manera crítica y reflexiva facilitan el aprendizaje, la aplicación y el uso de la ciencia, la tecnología y la innovación en diferentes grupos sociales; este programa, mediante un acercamiento pedagógico y participativo, fomenta la apropiación social del conocimiento y el uso de las herramientas de investigación, promoviendo el fortalecimiento de las líneas activas y campos de acción de la universidad, la articulación de las funciones universitarias, en un contexto de promoción de la cultura científica y creativa (Tirado y López, 2018).

En esta investigación se llevó a cabo una revisión sistemática de la literatura. Esto significa que se realizó un proceso estructurado de aproximación a la literatura sobre "estrategias pedagógicas para el fomento de la formación de capacidades en CTel", fundamentado en una búsqueda integral de artículos en bases de datos electrónicas y herramientas bibliográficas.

Específicamente, la revisión sistemática de la literatura se llevó a cabo en las herramientas: Google Scholar, Scopus y Web of Science. Esta elección se fundamentó en que estas herramientas abarcan la mayoría de la literatura relevante en el campo de estudio con el que se asocia esta investigación. Por último, se debe destacar que esta revisión se centró en contribuciones teóricas y empíricas producidas durante el periodo comprendido entre el año 1995 y el mes de abril de 2020. La elección de este periodo se basa en que a mediados de la década de los 90 el papel estratégico de las universidades, a partir de sus resultados de investigación, comenzó a destacarse (Koschatzky y Stahlecker, 2010). 


\section{Ecuación de búsqueda}

En las herramientas mencionadas para buscar artículos relevantes se ejecutó una búsqueda electrónica orientada por los términos claves que se derivan de los intereses temáticos de esta investigación. Adicional a esto se usaron combinaciones múltiples de los términos que se relacionan con el fenómeno de "formación de capacidades en ciencia, tecnología e innovación". En la siguiente lista se presentan las combinaciones de términos utilizadas en las herramientas bibliográficas. Es importante destacar que la ecuación fue ejecutada solamente en el idioma inglés.

- Pedagogical strategies in $\mathrm{CTel}$

- Effective teaching

- Opportunities for the CTel

- Strategic plan

- Teaching excellence and innovation

- Research pedagogical in higher education

- High impact teaching strategies

- Scientific research in higher education

\section{Resultados de la revisión sistemática} de la literatura y selección de los estudios

La aplicación de la ecuación de búsqueda generó como resultado 87 artículos. De este total, se eliminaron aquellos artículos que no tenían relación con la "formación de capacidades en CTel". Se excluyeron artículos que abordan temáticas que se desvían del fenómeno de estudio de esta investigación.

Finalmente, la revisión produjo un total de 26 artículos que guardan una relación directa con la "formación de capacidades en CTel en educación superior". 


\section{Procedimiento de análisis de los artículos}

\section{obtenidos a través de la revisión de la literatura}

Se realizó una lectura detallada de cada artículo. Luego, para dar forma al estado del arte, se extrajeron tres elementos claves de cada artículo: el propósito-objetivo principal, la metodología (en términos generales) y sus hallazgos más importantes. Estos tres elementos dan cuenta de las tendencias que, a nivel de investigación, posee la literatura analizada.

Así mismo, utilizando la herramienta Connected Papers se identificaron los 5 artículos más citados en el tema de interés en el ámbito mundial (figura 1):

- Cebrián, G. y Junyent, M. (2015). Competencies in Education for Sustainable Development: Exploring the Student Teachers' Views. Sustainability, 7, 2768-2786.

- Lozano, R., Barreiro-Gen, M., Lozano, F. J. y Sammalisto, K. (2019). Teaching sustainability in European higher education institutions: Assessing the connections between competences and pedagogical approaches. Sustainability, 11, 1602.

- Hallinger, P y Chatpinyakoop, C. (2019). A bibliometric review of research on higher education for sustainable development, 1998-2018. Sustainability, 11, 2401.

- Wilhelm, S., Förster, R. y Zimmermann, A. M. (2019). Implementing competence orientation: Towards constructively aligned education for sustainable development in university-level teaching-and-learning. Sustainability, 11, 1891.

- Wiek, A., Withycombe, L. y Redman, C. L. (2011). Key competencies in sustainability: A reference framework for academic program development. Sustainability Science, 6, 203-218. 
Figura 1. Artículos más citados en el tema y la red de autores más relevantes en el ámbito mundial.

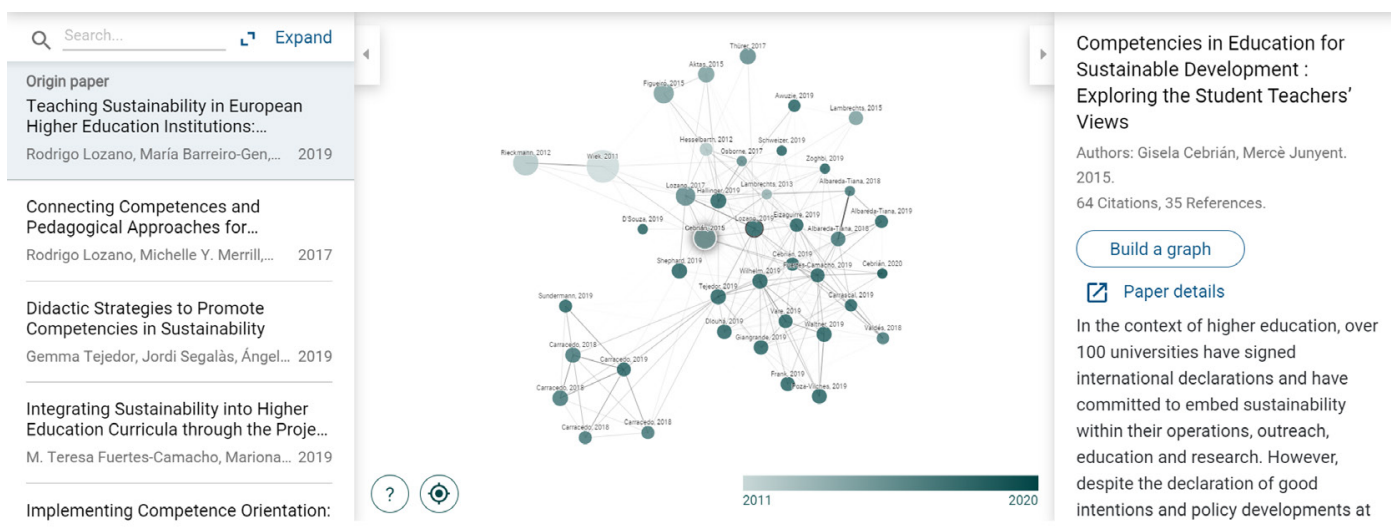

Fuente: construcción propia con el uso de la herramienta Connected Papers.

\section{Resultados}

El Programa de Formación de Capacidades en CTel surgió con el propósito de que la participación fuera más amplia y que permitiera incluir a egresados, docentes y administrativos. El programa permite de forma gradual la participación del total de los estudiantes a lo largo de su vida académica, fortaleciendo la articulación de las funciones universitarias, el trabajo con el sector productivo y la sociedad civil (Aparicio, Ostos, Cortés y Abadía, 2019).

Como resultado del proyecto, se desarrolló e implementó el Programa de Formación de Capacidades en CTel, integrado por ocho estrategias (figuras 2-3): 1) Grupos de estudio, 2) Grupos de trabajo, 3) Colectivos académicos, 4) Semilleros de investigación, 5) Programa institucional de jóvenes investigadores, 6) Programa institucional de jóvenes gestores, 7) Programa institucional de vocaciones científicas para niños, niñas, jóvenes y adolescentes, y 8) Trabajos de grado. 
Figura 2. Representación gráfica de las estrategias del Programa de Formación de Capacidades de la USTA y su articulación con las funciones universitarias.

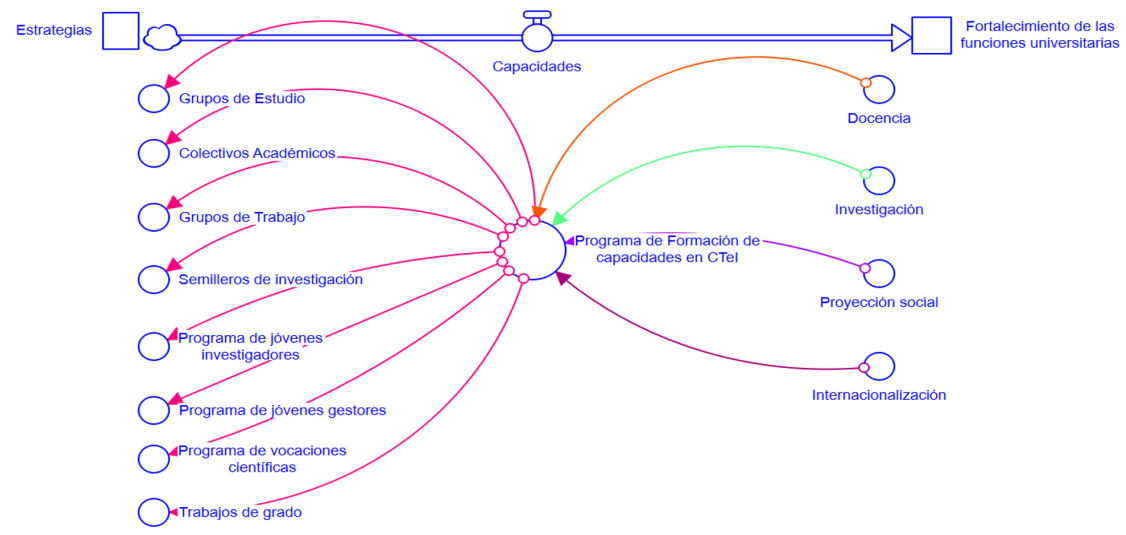

Fuente: elaboración propia.

Figura 3. Definición de las estrategias del Programa Institucional de Formación de Capacidades en CTel de la Universidad Santo Tomás.

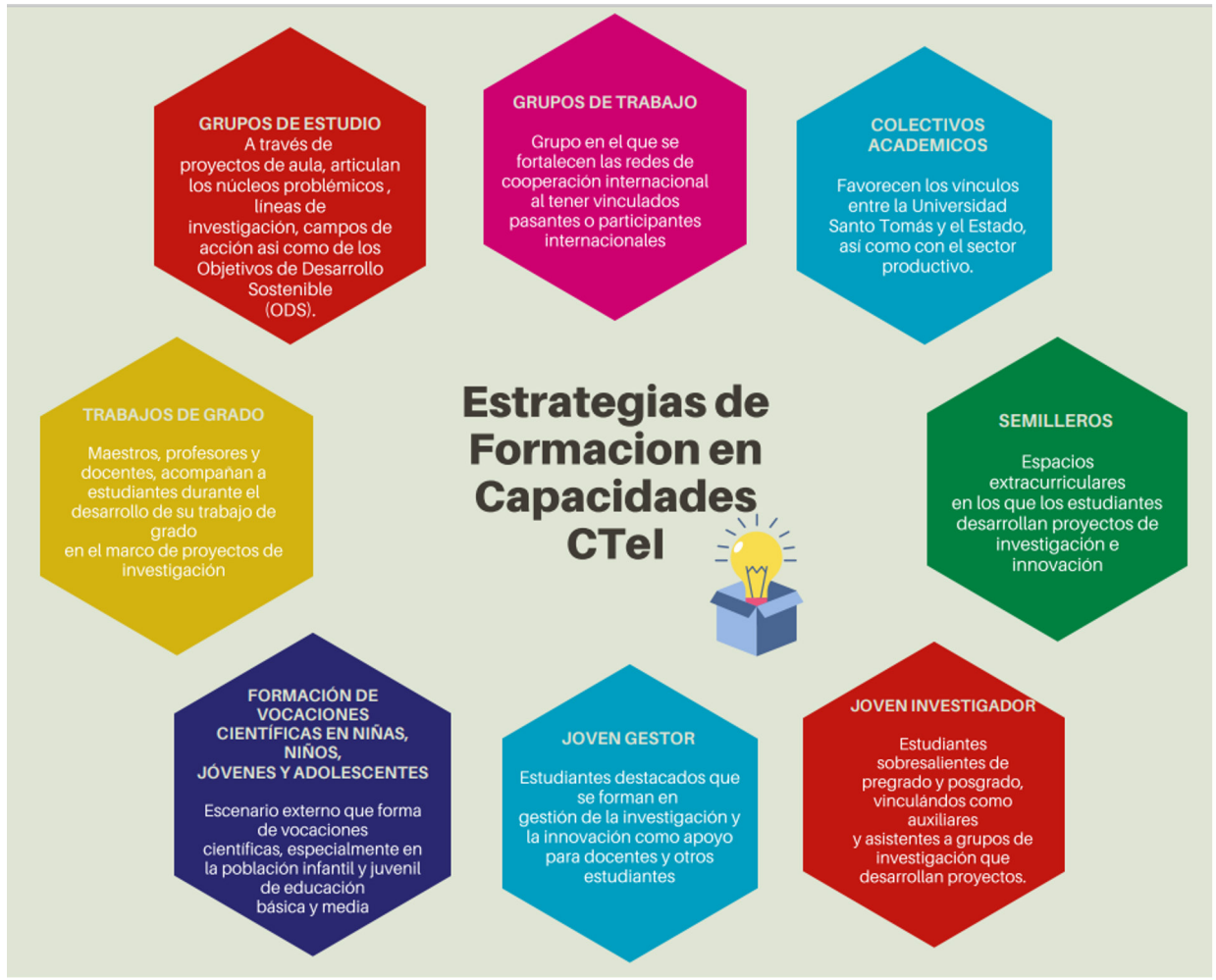

Fuente: elaboración propia. 


\section{Articulación docencia-investigación}

\section{Inicio de la carrera académica en investigación}

Grupos de estudio. Estrategia en la que participan estudiantes, docentes, egresados y administrativos con el interés de profundizar en un tema de investigación común, en el marco de los campos de acción de la USTA: sociedad y ambiente. La estrategia, fruto de la interacción de las funciones universitarias, permite la producción de working papers y material audiovisual que sintetiza la reflexión, transfiere socialmente el conocimiento y se comparte libremente al ser publicado en el repositorio institucional para garantizar su visibilidad e impacto en un escenario de ciencia abierta (OstosOrtiz y Aparicio-Gómez, 2020). La estrategia busca formar en la investigación a los participantes.

Semilleros de investigación. Estrategia en la cual docentes y estudiantes desarrollan en espacios extracurriculares proyectos de investigación e innovación (Pacheco Martínez, Suárez Acevedo, Ostos Ortiz, Silva Vivas y Marcucci Díaz, 2017; Quintero-Corzo, Munévar-Molina y Munévar-Quintero, 2008). La estrategia busca la articulación de los semilleros con las líneas de trabajo de los grupos de investigación.

\section{Fortalecimiento de capacidades en CTeI}

Jóvenes investigadores. En esta estrategia participan estudiantes y egresados destacados seleccionados a través de convocatoria, que aportan a los grupos de investigación en el desarrollo de sus proyectos como auxiliares y asistentes de investigación. Los estudiantes y/o egresados participantes reciben apoyo económico durante el tiempo de duración del proyecto (Ostos Ortiz, Londoño Bernal y Jiménez Zapata, 2020; Romero, 2007).

Jóvenes gestores de investigación e innovación. En esta estrategia participan a través de convocatoria estudiantes destacados, quienes en su proceso de formación son acompañados por la Dirección 
de Investigación en el desarrollo de habilidades en gestión de la investigación e innovación, reciben apoyo económico y contribuyen con el fortalecimiento de la cultura de la ciencia, la tecnología y la innovación en la USTA (Quevedo, 1999; Aparicio y Ostos, 2020; Ostos Ortiz, 2020).

Trabajos de grado. Estrategia de acompañamiento de estudiantes con docentes expertos para consolidar la formación en investigación a través de proyectos. Los lineamientos para esta estrategia se acogen a lo establecido en el apartado de Opciones de grado en el Reglamento Estudiantil (Mejía y Cely, 2014; Galán, Guillén y Mogollón, 2014).

\section{Articulación investigación-internacionalización}

Grupos de trabajo. Esta estrategia involucra estudiantes, docentes, egresados, administrativos e invitados internacionales; permite formar y fortalecer redes académicas y de cooperación en investigación, así como la construcción de centros de pensamiento en torno a los campos de acción de la USTA (Ostos-Ortiz y Cortés-GaIlego, 2019). La estrategia fomenta la cooperación académica e investigativa a nivel nacional e internacional. Este escenario fomenta la cooperación con investigadores y centros de investigación internacionales para favorecer el trabajo inter- y transdisciplinar con grupos y centros de investigación de todo el mundo.

\section{Articulación investigación-proyección social}

Colectivos académicos. En esta estrategia de formación se vinculan los estudiantes, docentes y egresados, en representación de la Universidad, con el sector productivo y/o la sociedad civil para el desarrollo de retos de innovación con el propósito de transformar realidades sociales, generando producción académica que aporta a la solución de problemas socialmente relevantes (Aparicio y Ostos, 2020; Ostos Ortiz, 2020; Ostos-Ortiz y Aparicio-Gómez, 2020). 
La estrategia fomenta la relación entre universidad, sector productivo y sociedad.

Esta estrategia busca que la universidad establezca alianzas con la sociedad civil y el sector productivo para resolver problemas reales a través de desarrollo de proyectos de investigación e innovación.

\section{Articulación educación básica y media con la educación superior}

Vocaciones científicas. Esta estrategia vincula estudiantes de nivel de educación básica y media con estudiantes de pregrado, posgrado y docentes. Es un programa para la formación de vocaciones para la investigación con el objetivo de transformar la cultura de la ciencia, la tecnología y la innovación en el país (Noronha y Ambiel, 2006). Esta estrategia enriquece la relación entre la media vocacional y la universidad.

En la tabla 1 se presentan las estrategias incluidas en el Programa de Formación de Capacidades en CTel de la USTA.

Tabla 1. Estrategias incluidas en el Programa de Formación de Capacidades en CTel de la USTA.

\begin{tabular}{|c|c|c|}
\hline Estrategias & Participantes & $\begin{array}{l}\text { Producción } \\
\text { académica }\end{array}$ \\
\hline Grupos de estudio & $\begin{array}{l}\text { Esta estrategia involucra a estudiantes, } \\
\text { egresados, administrativos, que acompañados } \\
\text { por uno o más docentes desarrollan proyectos } \\
\text { de aula, profundizando en un tema de interés } \\
\text { común. }\end{array}$ & $\begin{array}{l}\text { - Working paper } \\
\text { - Material audiovisual } \\
\text { - Artículos de revisión } \\
\text { de tema }\end{array}$ \\
\hline Grupo de trabajo & $\begin{array}{l}\text { Esta estrategia involucra a estudiantes, } \\
\text { egresados, invitados internacionales, y } \\
\text { docentes que trabajan en redes académicas de } \\
\text { cooperación internacional y constituyen la base } \\
\text { para la creación de centros de pensamiento. }\end{array}$ & $\begin{array}{l}\text { - Working paper } \\
\text { - Material audiovisual } \\
\text { - Artículos producto de } \\
\text { la investigación } \\
\text { - Innovación y } \\
\text { desarrollo tecnológico }\end{array}$ \\
\hline Colectivos académicos & $\begin{array}{l}\text { Esta estrategia involucra estudiantes y docentes } \\
\text { que en vínculo con el sector público y productivo } \\
\text { desarrollan retos de innovación para la } \\
\text { transformación de realidades sociales. }\end{array}$ & $\begin{array}{l}\text { - Productos de } \\
\text { nuevo conocimiento, } \\
\text { innovación, divulgación } \\
\text { y apropiación social del } \\
\text { conocimiento }\end{array}$ \\
\hline
\end{tabular}




\begin{tabular}{|c|c|c|}
\hline Estrategias & Participantes & $\begin{array}{l}\text { Producción } \\
\text { académica }\end{array}$ \\
\hline $\begin{array}{l}\text { Semilleros de } \\
\text { investigación }\end{array}$ & $\begin{array}{l}\text { Esta estrategia involucra a estudiantes y } \\
\text { docentes para el desarrollo de proyectos de } \\
\text { investigación e innovación. }\end{array}$ & $\begin{array}{l}\text { - Productos de } \\
\text { nuevo conocimiento, } \\
\text { innovación, divulgación } \\
\text { y apropiación social del } \\
\text { conocimiento }\end{array}$ \\
\hline $\begin{array}{l}\text { Programa de jóvenes } \\
\text { investigadores }\end{array}$ & $\begin{array}{l}\text { Esta estrategia involucra a estudiantes y } \\
\text { egresados sobresalientes acompañados por } \\
\text { docentes tutores. Fomenta la participación } \\
\text { de estudiantes en programas de maestría y } \\
\text { doctorado; los estudiantes y egresados reciben } \\
\text { un apoyo económico mensual durante el tiempo } \\
\text { de duración del proyecto. }\end{array}$ & $\begin{array}{l}\text { - Productos de } \\
\text { nuevo conocimiento, } \\
\text { innovación, divulgación } \\
\text { y apropiación social del } \\
\text { conocimiento }\end{array}$ \\
\hline $\begin{array}{l}\text { Programa de } \\
\text { jóvenes gestores de } \\
\text { investigación }\end{array}$ & $\begin{array}{l}\text { Esta estrategia involucra a estudiantes y } \\
\text { egresados sobresalientes que, acompañados } \\
\text { por la Dirección Nacional de Investigación e } \\
\text { Innovación, aprenden elementos de la gestión } \\
\text { de la investigación y la innovación, herramientas } \\
\text { del Sistema Nacional de Ciencia y Tecnología, } \\
\text { producción académica, gestores bibliográficos, } \\
\text { ética, bioética e integridad científica, entre otros } \\
\text { temas. Reciben apoyo económico. }\end{array}$ & $\begin{array}{l}\text { - Productos de } \\
\text { nuevo conocimiento, } \\
\text { innovación, divulgación } \\
\text { y apropiación social del } \\
\text { conocimiento. } \\
\text { - Productos } \\
\text { relacionados con } \\
\text { la gestión de la } \\
\text { producción académica } \\
\text { y la innovación }\end{array}$ \\
\hline $\begin{array}{l}\text { Programa de } \\
\text { vocaciones científicas }\end{array}$ & $\begin{array}{l}\text { Esta estrategia involucra a estudiantes, } \\
\text { docentes que en vínculo con el sector público y } \\
\text { productivo desarrollan retos de innovación para } \\
\text { la transformación de realidades sociales. }\end{array}$ & $\begin{array}{l}\text { - Productos de } \\
\text { apropiación social } \\
\text { del conocimiento } \\
\text { y estrategias } \\
\text { pedagógicas para el } \\
\text { fomento de la CTel }\end{array}$ \\
\hline Trabajos de grado & $\begin{array}{l}\text { Los desarrollan los estudiantes acompañados } \\
\text { por docentes; directores y codirectores como } \\
\text { opción para obtener su título profesional o de } \\
\text { posgrado; los lineamientos se establecen en el } \\
\text { Reglamento Estudiantil. }\end{array}$ & $\begin{array}{l}\text { - Productos de } \\
\text { nuevo conocimiento, } \\
\text { innovación, divulgación } \\
\text { y apropiación social del } \\
\text { conocimiento }\end{array}$ \\
\hline
\end{tabular}

Fuente: elaboración propia.

Las ocho estrategias de formación de capacidades en CTel se registran ante la Dirección de Investigación e Innovación, que realiza acompañamiento, y al final del semestre se reciben y revisan los productos académicos como resultado del ejercicio; además, se visibilizan y certifican para obtener impacto académico (Universidad Santo Tomás - Dirección de Investigación e Innovación, 2019).

Tal como se ha descrito en apartados anteriores, antes del 2019, año de implementación del Programa de Formación en Capacidades en $\mathrm{CTel}$, la estrategia preponderante eran los semilleros de investigación. Sin embargo, en el primer semestre de 2019 se inició el Programa Institucional de Formación de Capacidades en CTel 
con una participación de 35 grupos de estudio, 3 grupos de trabajo, 12 colectivos académicos, 70 semilleros de investigación, 33 jóvenes investigadores y 12 jóvenes gestores seleccionados por convocatoria. Así mismo, se registraron 24 docentes participando de la estrategia de vocaciones científicas con el Programa Ondas de Colciencias (Corchuelo-Rodríguez, 2017).

Estas cifras muestran la acogida que tuvieron estas estrategias en la universidad. Los semilleros de investigación continuaron trabajando y las cifras de las nuevas estrategias muestran una importante acogida del programa entre docentes, administrativos, estudiantes, egresados, sector público y productivo en cooperación con la USTA, que ahora hacen parte del Sistema Institucional de Investigación e Innovación (Universidad Santo Tomás - Dirección de Investigación e Innovación, 2019).

Para el primer semestre del año 2020, fueron registrados ante la Dirección de Investigación e Innovación: 67 grupos de estudio, 11 grupos de trabajo, 13 colectivos académicos, 88 semilleros de investigación, 34 jóvenes investigadores y 2 programas de vocaciones científicas con cooperación nacional e internacional. En las figuras 4-6 se presentan los datos de participación en las estrategias implementadas a través del Programa de Formación de Capacidades en CTel en la USTA desde el primer semestre de 2019.

Figura 4. Número de estudiantes participantes de las estrategias en el primer semestre de 2019.

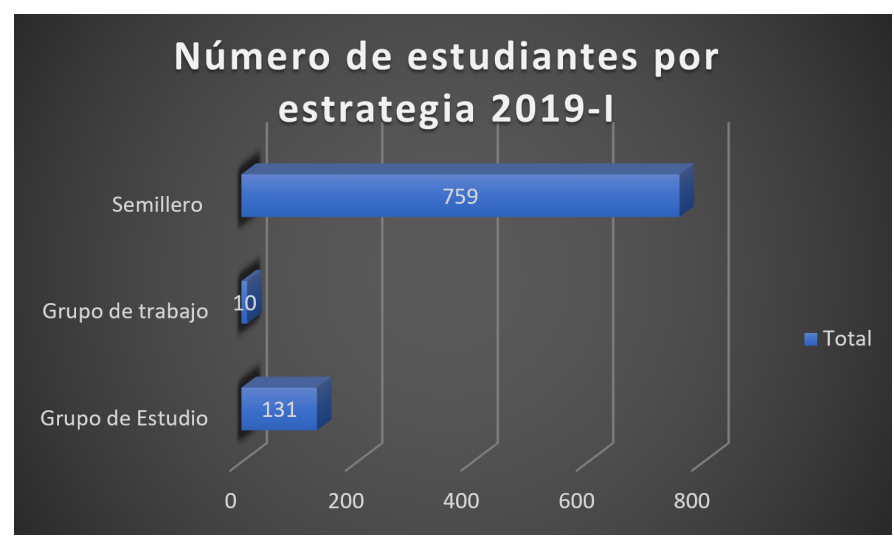

Fuente: elaboración propia. 
Figura 5. Número de estudiantes participantes de las estrategias en el segundo semestre de 2019.

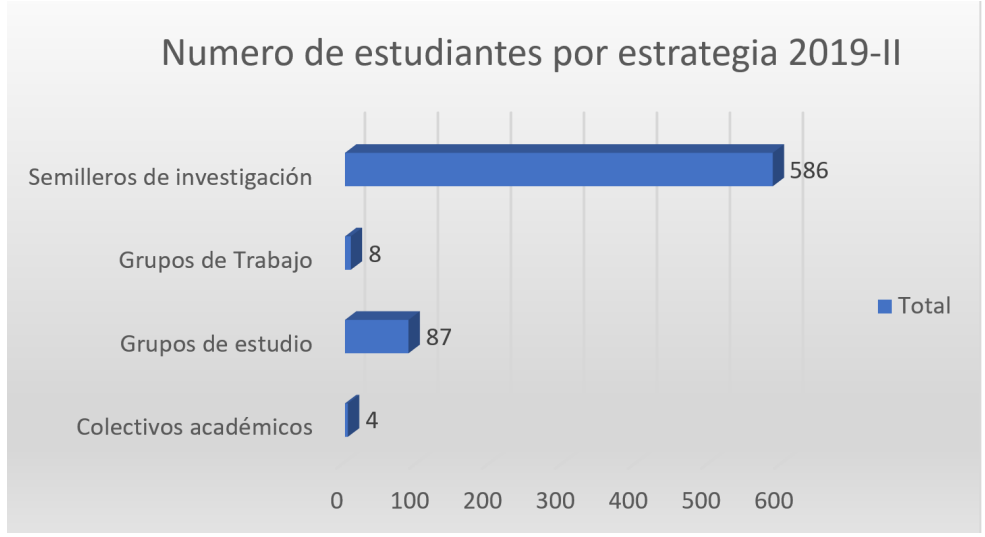

Fuente: elaboración propia.

Figura 6. Número de estudiantes participantes de las estrategias en el primer semestre de 2020.

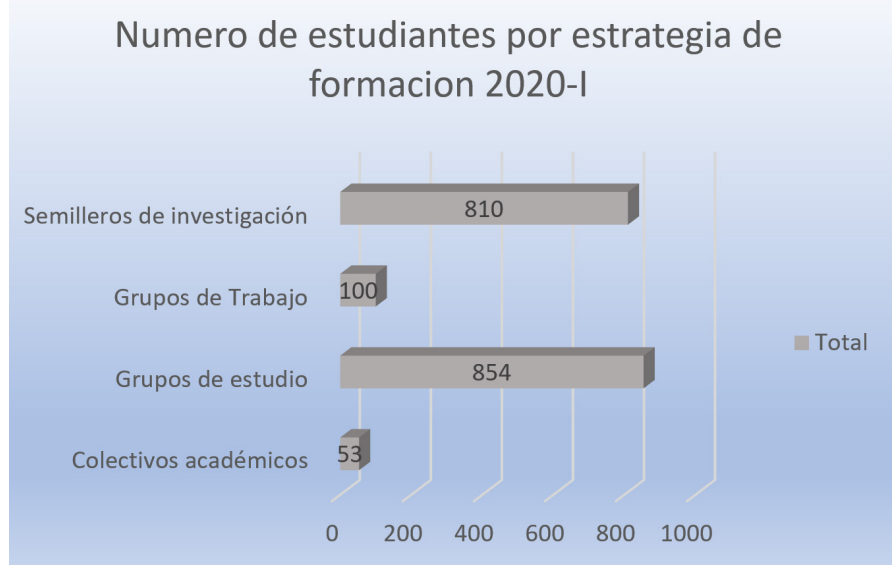

Fuente: elaboración propia.

En el segundo año de la implementación de las estrategias de formación los resultados han sido positivos y se ha alcanzado el objetivo propuesto, que es incentivar la participación y vinculación de los estudiantes apropiando la cultura de la investigación y la innovación.

Así mismo, es importante resaltar que las estrategias implementadas se articulan con los campos de acción definidos para la USTA, 
que se definen como "áreas o espacios de realidad significativa, con límites relativos, donde convergen las funciones sustantivas a partir de procesos investigativos rigurosos, en relación con las problemáticas que han sido identificadas y que son de interés para la USTA, con evidencia de contexto, experiencia e interdisciplinariedad". En este contexto, se realizó un estudio de vigilancia tecnológica que permitió identificar dos grandes campos de acción; sociedad y ambiente, que se articulan con los Objetivos de Desarrollo Sostenible (ODS) (Ostos-Ortiz y Cortés-Gallego, 2019).

\section{Discusión}

El programa implementado en la Universidad Santo Tomás es innovador, puesto que durante años el único espacio extracurricular para la formación de capacidades en ciencia, tecnología e innovación establecido en instituciones de educación superior en Colombia han sido los semilleros de investigación (Aparicio, Ostos, Cortés y Abadía, 2019). Actualmente, con estas nuevas estrategias que integran el programa se abre un abanico de posibilidades para que estudiantes, docentes y egresados, en conjunto con el sector productivo y la sociedad civil, puedan vincularse y participar de estos espacios de formación que buscan fortalecer la cultura CTel en la USTA y contribuir a la apropiación de la misma en el país (Quintero-Corzo, Munévar-Molina y Munévar-Quintero, 2008).

Los estudiantes y egresados se vinculan de forma voluntaria al programa y esto ha permitido la generación de nuevas alianzas y convenios con el sector público y productivo, lo cual ha generado productos de desarrollo tecnológico e innovación (Aparicio y Ostos, 2020), así como productos de nuevo conocimiento que aportan a la solución de problemas socialmente relevantes.

El Programa de Formación de Capacidades en CTel ha permitido generar nuevo conocimiento, avanzar en desarrollo tecnológico, promover la formación de recurso humano y contribuir a la apropiación social del conocimiento, así como la generación redes aca- 
démicas y de cooperación con el sector público y productivo del país (Tirado y López, 2018; Colciencias, 2018).

El programa se inició en la Sede Bogotá, y en el año 2020 se realizó su adaptación y normalización en todas las sedes, seccionales y centros de atención universitaria (CAU) de la Universidad Santo Tomás, fortaleciendo y promoviendo su carácter multicampus y los valores institucionales.

Para apropiar el Programa de Formación de Capacidades en CTel se han realizado jornadas de capacitación permanente para docentes en todas las divisiones académicas, facultades, departamentos e institutos de la USTA. También se capacitaron los líderes de investigación de la universidad y a los comités de investigación de cada programa, que transfieren la información a estudiantes y egresados.

De la misma forma, se han realizado capacitaciones con los estudiantes en cada inicio de semestre, motivando su participación y adhesión al programa. Adicionalmente, se desarrollaron iniciativas publicitarias; e información en la web, redes sociales, pendones, boletines divulgativos, difusión masiva en correos institucionales, entre otras.

Mediante el análisis y seguimiento permanente del programa se espera fortalecer las redes de investigación institucional, la participación en redes de investigación y promover la cooperación con grupos y centros de investigación en todo el mundo, favoreciendo la inter- $y$ transdisciplinariedad en el abordaje de problemas complejos. 


\section{Referencias bibliográficas}

Aparicio, O. y Ostos, O. (eds.). (2020). Innovación educativa y gestión del conocimiento. Bogotá: Universidad Santo Tomás.

Aparicio, O., Ostos, O., Cortés, M. y Abadía, C. (2019). Análisis sistemático de los artículos publicados en la Revista Interamericana de Educación, Investigación y Pedagogía (RIIEP) durante 2014 a 2019. Revista Interamericana de Investigación, Educación y Pedagogía (RIIEP), 12(2), 225-240.

Colciencias (2018). Libro Verde 2030: Política Nacional de Ciencia e Innovación para el Desarrollo Sostenible. Bogotá: Colciencias.

Corchuelo Rodríguez, C. (2017). Estrategias de visibilidad de la producción científica y académica de los grupos de investigación de la Universidad Santo Tomás. Recuperado de https://repository.usta.edu. co/handle/11634/9806

Galán, L., Guillén, S. y Mogollón, E. (2014). Acompañamiento estudiantil, una experiencia de intervención en el programa de apoyo integral al estudiante de la división de ingenierías de la Universidad Santo Tomás (USTA). Artículo presentado en la Cuarta Conferencia sobre el Abandono en la Educación Superior (Clabes). Recuperado de https://revistas.utp. ac.pa/index.php/clabes/article/view/1026

Koschatzky, K. y Stahlecker, T. (2010). New forms of strategic research collaboration between firms and universities in the German research system. International Journal of Technology Transfer and Commercialisation, 9(1-2), 94-110.

Mejía, B y Cely, D. (2014). Proyecto de formación pedagógica y didáctica a monitores académicos: propuesta de acompañamiento a los estudiantes de la Universidad Santo Tomás. Artículo presentado en la Cuarta Conferencia sobre el Abandono en la Educación Superior (Clabes). Recuperado de https://revistas.utp.ac.pa/index.php/clabes/ article/view/1079 
Noronha, A. P. P. y Ambiel, R. A. M. (2006). Orientação profissional e vocacional: análise da produção científica. Psico-USF, 11(1), 75-84.

Ostos-Ortiz, O. L. y González Gil, E. (2020) Gestión del conocimiento, un reto en la educación superior. En O. Aparicio y O. Ostos-Ortiz (eds.), Innovación educativa y gestión del conocimiento (pp. 19-42). Bogotá: Universidad Santo Tomás.

Ostos-Ortiz, O. y Cortés-Gallego, M. (2019). Los campos de acción en la Universidad Santo Tomás: resultados del estudio 2012-2018. Revista Interamericana de Investigación, Educación y Pedagogía (RIIEP), 12(1), 15-40.

Ostos-Ortiz, O. L. y Aparicio-Gómez, O. Y. (2020). Investigación, innovación y transferencia del conocimiento. Working Paper n.o 197503. Universidad Santo Tomás.

Ostos-Ortiz, O. L., Londoño Bernal, N. y Jiménez Zapata, M. A. (2020). Bienvenida Jóvenes Investigadores 2020.

Pacheco Martínez, J. A., Suárez Acevedo, C., Ostos Ortiz, O. L., Silva Vivas, J. P. y Marcucci Díaz, C. E. (eds.) (2017). Portafolio Semilleros de Investigación (sede principal). Universidad Santo Tomás - Vicerrectoría Académica General.

Quevedo, M. (1999). La gestión en la investigación universitaria. Innovar, 13, 15-32.

Quintero-Corzo, J., Munévar-Molina, A. M. Munévar-Quintero, F. I. (2008). Semilleros de investigación: una estrategia para la formación de investigadores.

Ramírez Martínez, J. E., Silva Vivas, J. P., Hernández Merchán, M. A., López Pachón, C. P. y Ostos Ortiz, O. L. (2018). Formación de capacidades en ciencia, tecnología e innovación. Recuperado de https://repository. usta.edu.co/handle/11634/13720 
Romero, M. N. (2007). Jóvenes investigadores: la formación del ser investigador, obstáculos y desafíos. Index de Enfermería, 16(57), 50-54.

Tirado, Ly López, L. (2018). El Libro verde de Colciencias: transformaciones sociales, económicas e incidencias de las políticas científicas en Colombia. Kairós Gerencial, 3(6).

Universidad Santo Tomás. (2004). Proyecto Educativo Institucional. Bogotá: Universidad Santo Tomás.

Universidad Santo Tomás. (2018). Estatuto Orgánico.

Universidad Santo Tomás - Dirección de Investigación e Innovación. (2019). Política de Investigación e Innovación, Creación Artística y Cultural de la Universidad Santo Tomás. Recuperado de https:// repository.usta.edu.co/handle/11634/19695 